\title{
Platelet-rich plasma versus lidocaine as tenotomy adjuvants in people with elbow epicondylopathy: a randomized controlled trial
}

Jose Ignacio Martin 1,2, Leire Atilano ${ }^{1,2}$, Josu Merino 2,3, Igor Gonzalez ${ }^{2,3}$, Gotzon Iglesias ${ }^{1,2}$, Luis Areizaga ${ }^{2,3}$, Paola Bully ${ }^{4}$, Gonzalo Grandes ${ }^{4}$ and Isabel Andia ${ }^{2 *}$ (D)

\begin{abstract}
Objectives: To determine the efficacy of platelet-rich plasma (PRP) compared to lidocaine as a tenotomy adjuvant for people with elbow tendinopathy.

Methods: Our study was a parallel-group, double-blind, randomized trial involving 71 patients with recalcitrant elbow tendinopathy who received two sessions of ultrasound-guided tenotomy with either PRP or lidocaine in a tertiary public hospital. The primary end point was the percentage of patients with an improvement exceeding 25\% reduction in disability (Spanish version of the Disabilities of the Arm, Shoulder and Hand questionnaires-DASH-E) at 6 and 12 months; the secondary outcome was the percentage of patients exceeding 25\% reduction in pain (VAS-P).

Results: There was no evidence of significant differences in the proportion of patients who experienced clinically relevant improvements. After 6 months, 18 patients (78.59\%) in the lidocaine group and 19 patients (73.08\%) in the PRP group showed improved function above $25 \%$ (unadjusted odds ratio, 0.90; 95\% confidence interval [CI], 0.90 (0. 17 to 4.60$)$ ); 21 patients (72.21\%) in the lidocaine group versus 22 patients (84.62\%) in the PRP group achieved more than $25 \%$ pain reduction (unadjusted odds ratio, $0.48 ; 95 \% \mathrm{Cl}, 0.10$ to 2.37 ). After 12 months, 17 patients (70.83\%) in the lidocaine group versus 19 patients (76\%) in the PRP group had improved function (unadjusted odds ratio, 0.71; 95\% Cl, 0.13 to 3.84), and 19 patients (76\%) in the lidocaine group versus 20 patients (90.91\%) in the PRP group had improved pain above $25 \%$ (unadjusted odds ratio, $0.35 ; 95 \% \mathrm{Cl}, 0.06$ to 2.51 ). Hypercholesterolemia and baseline vascularization influenced outcomes. There were no differences between groups in the adjusted odds ratios.
\end{abstract}

Conclusion: PRP results in similar improvements to those obtained with lidocaine. Selecting patients according to their pretreatment status can improve treatment efficacy.

Trial registration: NCT01945528, EudraCT 2013-000478-32. Registered 18 August 2013, enrolment of the first participant 10 March 2014

Keywords: Elbow tendinopathy, Epicondylitis, Lateral, Medial, Tenotomy, Platelet-rich plasma, Function, Pain, Randomized controlled study, Sonography

\footnotetext{
*Correspondence: isabel.andiaortiz@osakidetza.eus; iandia2010@hotmail.com

${ }^{2}$ Regenerative Medicine, BioCruces Health Research Institute, Cruces

University Hospital, 48903 Barakaldo, Spain

Full list of author information is available at the end of the article
}

(c) The Author(s). 2019 Open Access This article is distributed under the terms of the Creative Commons Attribution 4.0 International License (http://creativecommons.org/licenses/by/4.0/), which permits unrestricted use, distribution, and reproduction in any medium, provided you give appropriate credit to the original author(s) and the source, provide a link to the Creative Commons license, and indicate if changes were made. The Creative Commons Public Domain Dedication waiver (http://creativecommons.org/publicdomain/zero/1.0/) applies to the data made available in this article, unless otherwise stated. 


\section{Introduction}

Elbow tendinopathy (epicondylalgia) is the most common tendinopathy in upper limbs and it has a relevant economic burden [1]. It can affect the wrist extensors that originate from the lateral epicondyle and/or the flexors originating from the medial epicondyle, with similar rates between men and women. Lateral epicondylopathy is more prevalent than medial epicondylopathy, i.e., $1.3 \%$ versus $0.4 \%$ [2], but the prevalence could be as high as $5.2 \%$ or higher in middle-aged people performing repetitive movements and/or forceful tasks as part of their working activities [3, 4].

Local injection treatments are proposed as a cost-effective conservative management in patients who are recalcitrant to bracing, non-steroidal anti-inflammatory drugs (NSAIDs), or physiotherapy before considering tendon release through arthroscopy or open surgery. The most common injectable is corticosteroids, a palliative treatment frequently administered on the peritendon. Actually, the target could be sympathetic and sensory innervation could be found in the superficial side of the extensor carpi radialis brevis (ECRB) origin [5]. However, in persistent elbow tendinopathy, corticosteroid injections delayed complete recovery and increased the recurrence rate [6]; therefore, research has shifted towards the development of tendon regenerative treatments.

Needle tenotomy is a commonly performed therapy that involves passing a needle through the abnormal tendon multiple times, with intensities that can vary according to the number of passes, ranging from 5 to 50 , with needle diameters ranging from 20 to 25 gauge (also described as fenestrations, needling, or peppering) [7]. Any type of tenotomy can be considered a regenerative technique because ensuing microtrauma induces a healing response through an early gene expression (transcription factors) pattern similar to mechanical loading, thereby enhancing tendon structure and strength [8]. The goal is to convert a chronic degenerative process into acute inflammation by breaking the vicious loop of failed healing and remodelling provoked by accumulated tendon damage [9]. Nevertheless, few controlled studies have examined the efficacy of percutaneous needle tenotomy for elbow epicondylopathy [10].

Moreover, tenotomy can be "dry" (as a standalone procedure) or can be part of a combined intervention; i.e., associated with anaesthetics, corticosteroids, or regenerative products, such as blood or platelet-rich plasma (PRP). PRP consists of a plasma preparation with a concentration of platelets above peripheral blood (pure PRP) and optionally with concentrated leukocytes (L-PRP). The molecular pool present in PRP modulates biological processes involved in tissue healing, including modulation of angiogenesis and inflammation as well as cell proliferation and survival [11]. In addition, it provides a fibrin template that can guide cells in the injured areas. Although these biological actions have been shown in vitro [12], the clinical efficacy of PRP preparations in tendons is still controversial.

PRP has been widely used to treat diverse tendinopathies, e.g., rotator cuff, Achilles, patellar and gluteal tendons, but current meta-analyses have shown modest improvements [13-15]. Specifically, the value of PRP in epicondylopathy has been investigated in more than a dozen controlled trials [16], mostly using corticosteroids or whole blood as comparators. However, efficacy has not been demonstrated yet, in part due to heterogeneity among clinical studies that was mainly attributed to variability in PRP formulations [17] and procedures of delivery. In addition, whether PRP is more efficient in enthesopathies, such as epicondylopathy, or tendinopathies in the proper tendon is controversial [18].

Our objective was to examine whether pure PRP (with a moderate concentration of platelets) is a good adjuvant to tenotomy in the management of chronic elbow tendinopathy (medial and lateral). We have used [tenotomy + lidocaine] as a comparator, which is our gold standard. Consistent with the classic view of chronic wounds where a single intervention may be insufficient to regenerate a chronic injury, we performed two interventions with a 2-week interval.

\section{Methods}

We performed a parallel-group, assessor- and patientblinded, randomized controlled trial in a tertiary public hospital.

Inclusion criteria were tendinopathy present in either the lateral or medial elbow in patients who had failed conservative treatments. The latter consisted of 4-6 weeks of antialgic and anti-inflammatory medication (NSAIDs), physical therapy associated with orthosis and at least one corticosteroid infiltration in the painful area. Patients were included if they had symptoms lasting at least 3 months or longer and baseline elbow pain above 3/10 during resisted wrist extension or flexion, in the case of lateral or medial epicondylopathy respectively. Other inclusion criteria were age between 18 and 75 years, body mass index (BMI) between 20 and 35 and commitment to comply with all study procedures. The most symptomatic elbow was treated in bilateral patients. Patients suspended any analgesic therapy 15 days before the intervention; merely paracetamol $1 \mathrm{gr} / 6$ h or metamizole granulated oral suspension, maximum 4 g/day, was allowed. Oral corticoids were not permitted nor were NSAIDs for up to ten or more consecutive days.

The exclusion criteria were the presence of a full tendon tear; BMI > 35; systemic autoimmune rheumatologic disease (connective tissue diseases and systemic necrotizing vasculitis); poorly controlled diabetes mellitus (glycosylated haemoglobin above 9\%); blood disorders (thrombopathy, thrombocytopenia, anaemia with a $\mathrm{Hb}$ level $<9$ ); receiving 
immunosuppressive treatments; receiving local steroid injection within 3 months of randomization; receiving NSAIDs, opioids, or oral corticosteroids within 15 days before inclusion in the study; severe heart disease; patients unable to comply with scheduled visits due to work, or spending long periods away from their habitual residence; patients with active cancer or cancer diagnosed in the last 5 years; analytical diagnosis of hepatitis B or C or HIV infection; pregnant or lactating; and patients who were taking a drug in a clinical investigation. Initial patient selection was conditioned to the negative results in the analytical tests for hepatitis B or C or HIV infection.

The diagnosis was performed by orthopaedic surgeons and was merely clinical. Lateral epicondylopathy was diagnosed based on pain and maximum tenderness upon palpation at the common extensor origin at the lateral humeral epicondyle, pain upon resisted forearm supination, painful active wrist extension and painful passive wrist flexion. Medial epicondylopathy was diagnosed based on pain distal to the common flexor insertion, increased pain with resisted wrist flexion and pain with resisted forearm pronation and elbow extension. Patients with suspected ulnar neuropathy were not included. Clinical assessments were performed by the same orthopaedic surgeons that performed the inclusion assessment and were unaware of the treatment modality.

\section{Settings and locations}

The study protocol was approved by the local Ethics Committee of HUC, authorized by the Spanish Agency of Medicines (EudraCT 2013-000478-32), registered at clinicaltrials. gov (NCT01945528) and was published previously [19].

Between April 2014 and May 2017, a total of 85 patients with elbow tendinopathy were referred to the Orthopaedic Department at Cruces University Hospital, by general practitioners or by other orthopaedic departments, and they were assessed for eligibility. Eighty-two patients met the inclusion/exclusion criteria and underwent blood tests. All patients provided written informed consent and could decide to leave the study at any time.

\section{Interventions}

The experimental and control groups were ultrasound (US)-guided percutaneous tenotomy combined with PRP each alternate week for a total of two interventions and US-guided tenotomy combined with lidocaine each alternate week for a total of two interventions.

\section{PRP preparation}

Twenty-four $\mathrm{mL}$ of peripheral blood (i.e., three $9 \mathrm{~mL}$ tubes containing $0.9 \mathrm{~mL}$ of sodium citrate, Vacuette, Greiner BioOne, Switzerland) were withdrawn from all patients at every intervention. Pure (leukocyte-free) PRP was prepared by single spinning at $570 \mathrm{G}$ for $6 \mathrm{~min}$ and the plasma layer was collected, under laminar flow, avoiding aspirating the buffy coat, following our standard operating procedures. In doing so, we obtained approximately 6-8 $\mathrm{mL}$ of pure PRP (no leukocytes) without detectable leukocytes and a moderated enrichment of platelets (2.30 \pm 0.68 times above peripheral blood baseline). According to previous classifications [20], it can be described as pure platelet-rich plasma (P-PRP) or leukocyte-poor platelet-rich plasma, i.e., preparations without leukocytes and with a low-density fibrin network after activation.

At the interventional radiologist office, PRP is activated with $\mathrm{CaCl}_{2}$ (final concentration $22.5 \mathrm{mM}$ ) prior to loading $5 \mathrm{~mL}$ in a 10 - $\mathrm{mL}$ Luer-lock syringe.

\section{Tenotomy}

We performed all procedures with the patient in a supine position, the elbow flexed $120^{\circ}$ and forearm in pronation (lateral) or supination (medial), guided with a $4-13 \mathrm{MHz}$ high-frequency linear probe (Esaote MyLab 70 XVG, Esaote S.p.A. Genoa, Italy). A senior radiologist with more than 20 years of experience in musculoskeletal interventional ultrasonography performed all tenotomies.

A sterile protocol was followed as for any other musculoskeletal intervention. The subcutaneous tissues overlying the lateral epicondyle were infiltrated tangential to the plane of the lateral epicondyle with $2 \mathrm{~mL}$ lidocaine via a 22-gauge hypodermic needle. Then, the bulb containing the injectable (PRP or lidocaine) was connected to the needle, which was inserted parallel to the tendon long axis, from distal to proximal. The tendon was repeatedly fenestrated (15-25 times) by redirecting the needle in different directions, until softening of the tissue. In addition to piercing the tendon, the tip needle was used to abrade the periosteum. At the same time, the injectable was delivered in the areas of hypoechogenicity and the surround. A second intervention, involving approximately ten tendon perforations and no abrasions of the periosteum, was performed after 2 weeks. We injected $4.23 \pm 1.09 \mathrm{~mL}$ (range 1-5) of lidocaine and $4.47 \pm 1.11$ (range 1-5) of PRP in the first intervention and $4.18 \pm 1.14 \mathrm{~mL}$ (range 1-5) of lidocaine and $4.53 \pm 0.88 \mathrm{~mL}$ (range 2-5) of PRP in the second intervention. There were no differences between the injected volumes. After each intervention, patients were instructed to rest for the first $48 \mathrm{~h}$ and avoid weight lifting. Patients did not follow any post-procedural exercise programme, but they modified their activities and resumed physical work upon demand.

\section{Outcomes}

The primary outcome measures were the number (percent) of patients who achieved clinically relevant improvement, defined as a reduction in Disabilities of the Arm, Shoulder and Hand self-reported questionnaires, Spanish version (DASH-E), of at least $25 \%$ relative to baseline [21] 
at 6 and 12 months. Secondary outcome measures were the number (percent) of patients who achieved a clinically relevant improvement in pain, VAS-P (0-10 Likert scale), at 6 and 12 months. For safety assessments, patients recorded any adverse reaction and needed medication in a diary that was collected at each hospital visit. Acetaminophen was the only drug allowed for pain.

\section{Sample size}

We assumed that the relative improvement with the PRP intervention was 1.43, assuming that the differences between PRP and lidocaine would be similar to the differences reported with corticoids [22], and a patient loss of approximately $20 \%$. A sample size of 80 patients was expected to provide an $80 \%$ potency to detect any significant difference between the success rates in both groups $(\mathrm{P} 1=0.93$ and $\mathrm{P} 2=0.65)$ with a level of significance of $5 \%$, with each arm formed by 40 patients.

\section{Randomization and blinding}

An independent researcher performed randomization in blocks of four, using EPIDAT3.1, and created aluminium paper blinded envelopes with the numbered treatment allocation. The numbered envelopes were opened on the treatment day by the researcher who was in charge of the PRP preparation. All physicians (including orthopaedists involved in clinical outcome assessments and radiologists involved in ultrasound assessments), except one radiologist who performed the procedures, were unaware of treatment allocation. All patients were blinded to the treatment. Peripheral blood was drawn from all patients, and in each intervention, the syringe containing the treatment was wrapped with gauze hindering treatment visualization.

\section{Statistical methods}

To test the overall effect of the treatment, [tenotomy + PRP] and [tenotomy + lidocaine], on an intention-to-treat basis, we compared changes in function and pain levels between the two groups over 12 months. No imputation method was used to handle the missing data. To confirm comparability between groups at baseline, Student's $t$ test was used for continuous variables due to the proven normal distribution of the data in both groups, and a $x^{2}$ test was used for categorical variables. Spearman's tests were used to assess the association between outcome variables. Data are summarized as the mean and standard deviation for normally distributed variables, medians and interquartile ranges for non-normally distributed variables and the frequency and percentage for categorical variables. To evaluate the differences between the PRP and lidocaine groups, longitudinal generalized mixed models, with and without adjustments, were used, considering the repeated four follow-up measurements for each patient. The treatment, the time of measurement and treatment-bytime interaction were included as fixed effects in the models. Patients were included as random effects in the intercept of the different repeated measurements. Time evolution was considered as a categorical variable without autocorrelation within an individual. These options were chosen because they provided a better fit to our data. The overall effect of the treatment was assessed by testing the interaction between the treatment and time of measurement. Additionally, these models were also adjusted for baseline values of the outcome variables, socio-demographics and risk factors, possible determinants of pain and function. Likewise, to simplify the fixed effects structure, maximum likelihood ratio tests were used following backward, forward and stepwise strategies. Unadjusted and adjusted odds ratios with 95\% confidence intervals (CI) and mean differences with $95 \%$ CI were calculated for categorical and continuous outcomes, respectively. Planned contrasts were used to determine whether changes in the PRP group between baseline and each of the follow-up points were different from those observed in the lidocaine group. For all the contrasts, $p<$ 0.05 was considered the significance criterion.

A post hoc power calculation was performed based on longitudinal mixed effects models adjusted to the final sample size, actual data variability and clustering to detect differences with small (0.2 standard deviations), medium ( 0.5 standard deviations) and large ( 0.8 standard deviations) effect sizes between the comparison groups. All analyses were carried out using the SAS statistical package version 9.4.

\section{Results}

Figure 1 represents the flow of participants through the trial. Eighty patients, recruited between 2014 and 2017, meeting all inclusion criteria, were randomly allocated to the [tenotomy + PRP] and [tenotomy + lidocaine] groups. Four patients in the PRP group and one patient in the lidocaine group withdrew from the study before treatment for the following reasons: horse accident (1), preferred the surgical option (1), entitled to hip surgery (1), had no pain the day of the first intervention (1) and declined without any reason (1). One patient in the PRP group and three in the lidocaine group received only the first tenotomy and declined to receive the second intervention because they found the procedure too painful. A total of 71 patients were treated. During the study, one patient treated with PRP was lost to follow-up after the 6-week assessment and four patients (three from the lidocaine group and one from the PRP group) were lost after the 6-month assessment. We had additional missing data for two main reasons. First, many patients did not fill out the DASH-E properly (i.e., less than 25 answers over 30 questions, making the DASH-E invalid for analyses). Second, some patients attended the radiology service during follow-up but failed 


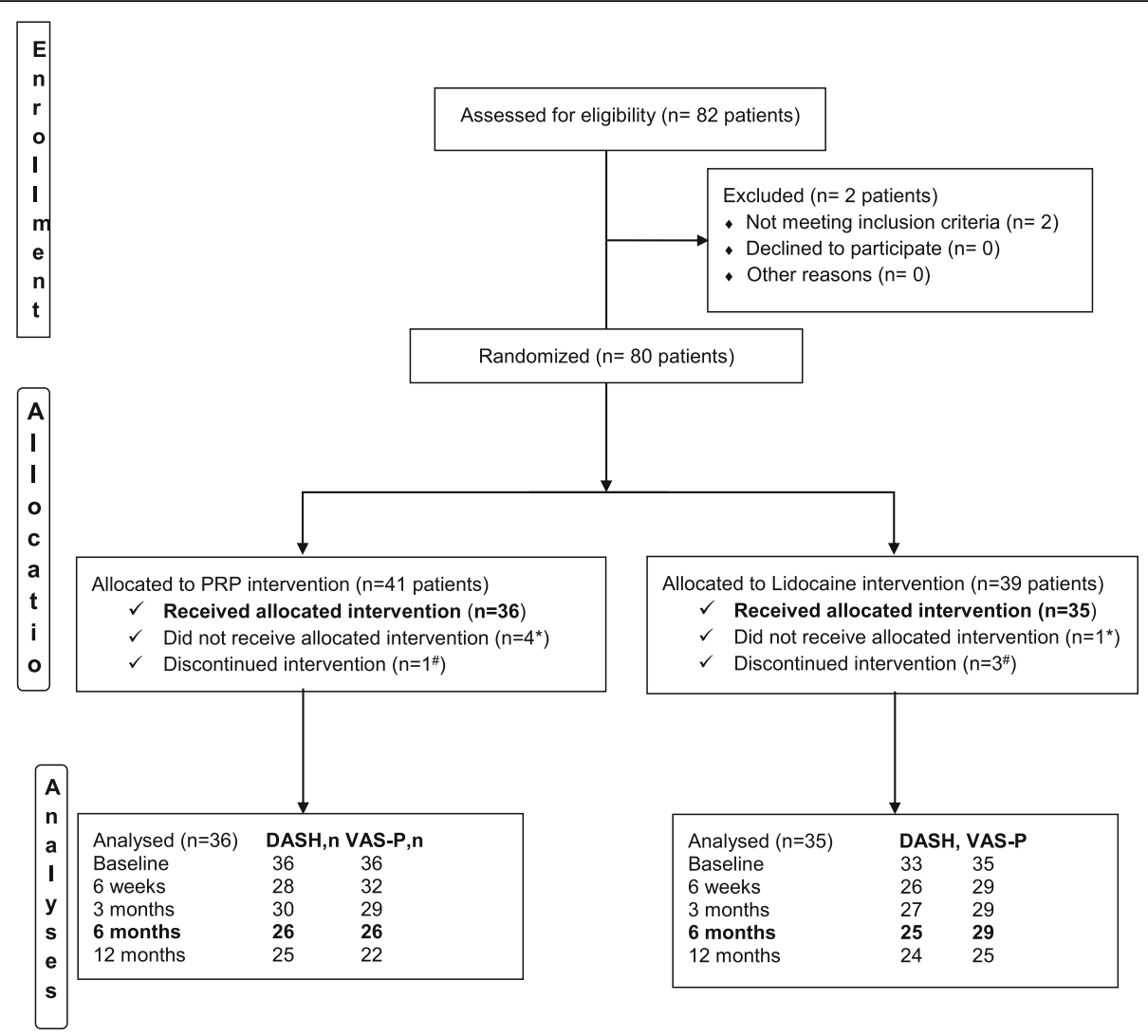

Fig. 1 Participants' flow diagram. Asterisk denotes did not receive allocated intervention because of: a horse accident (1), preferred the surgical option (1), entitled for hip surgery (1), had no pain the day of the first intervention (1) and declined without any reason (1). Number sign indicates discontinued intervention because of post-tenotomy pain. During the study, one patient treated with PRP was lost to follow-up after the 6-week assessment and four patients (three from the lidocaine group and one from the PRP group) were lost after the 6-month assessment. We had additional missing data for two main reasons. First, many patients did not fill the DASH properly (i.e., with less than 25 answers over 30 questions, the DASH was not valid for analyses). Second, some patients attended to the echography service during follow-up but failed orthopaedists 'consultation where clinical data were collected

to complete orthopaedist consultations where clinical data were collected. Sociodemographic and clinical characteristics were well-balanced at baseline (shown in Table 1). The association between pain and function increased throughout the follow-up in a similar way for both groups (see Table 2).

\section{Primary and secondary clinical outcomes}

Successful treatment was defined as more than $25 \%$ reduction in DASH-E or VAS-P scores after 1 year. Table 3 shows the rate of patients who met these criteria in each measurement. A high rate of patients showed enhanced function and pain reduction over the 12-month follow-up.

Data for functional improvement were available for 25 (71.43\%) and 24 (68.57\%) patients in the lidocaine group and $26(72.22 \%)$ and $25(69.44 \%)$ patients in the PRP group, at 6 and 12 months, respectively. There were no significant differences between therapies in terms of the rate of patients who achieved the minimum clinically important difference in function recovery at 6 or 12 months after treatment (Table 3). The differences in the percentage of success between [tenotomy+lidocaine] and the [tenotomy+PRP] were $-1.94 \%(95 \% \mathrm{CI},-30.46$ to 26.59$)$ at 6 months and $-6.03 \%(95 \% \mathrm{CI},-35.98$ to 23.90$)$ at 12 months.

In the case of pain, the frequencies for the available data were $29(82.85 \%)$ and $25(71.43 \%)$ in the lidocaine group and $26(72.22 \%)$ and $22(61.11 \%)$ in the PRP group. Likewise, there were no differences between tenotomy adjuvants (lidocaine versus PRP) in the rate of patients who achieved clinically important pain relief and differences in the percentage of success at 6 months, $-10.56 \%(95 \% \mathrm{CI}$, -33.01 to 11.89 ), and at 12 months, $-11.51 \%$ (95\% CI, 32.29 to 9.27). The differences between the two groups in the short term were not statistically significant either. The difference in the percentage of patients with better functionality at 6 weeks was $7.53 \%$ (95\% CI, -27.62 to 42.67$)$ and at 3 months was $26.62 \%$ (95\% CI, -0.82 to 54.06 ). The difference in the percentage of patients with pain reduction exceeding $25 \%$ at 6 weeks was $21.70 \%$ (95\% CI, - 
Table 1 Baseline sociodemographic clinical and sonographic characteristics in both groups

\begin{tabular}{|c|c|c|c|c|}
\hline \multirow{2}{*}{$\begin{array}{l}\text { Variable } \\
\text { Age, } n, M \pm S D\end{array}$} & \multicolumn{2}{|c|}{ Lidocaine $(N=35)$} & \multicolumn{2}{|c|}{$\operatorname{PRP}(N=36)$} \\
\hline & 35 & $48.26 \pm 7.64$ & 36 & $50.80 \pm 6.73$ \\
\hline \multicolumn{5}{|l|}{ Gender, no. (\%) } \\
\hline Male & 19 & $(54.3)$ & 14 & $(38.9)$ \\
\hline Female & 16 & $(45.7)$ & 22 & $(61.1)$ \\
\hline \multicolumn{5}{|l|}{ Manual worker, no. (\%) } \\
\hline No & 10 & $(29.4)$ & 11 & $(28.2)$ \\
\hline Yes & 24 & $(70.6)$ & 25 & $(71.8)$ \\
\hline \multicolumn{5}{|l|}{ Throwing sports, no. (\%) } \\
\hline No & 29 & $(87.9)$ & 33 & $(94.3)$ \\
\hline Yes & 4 & $(12.1)$ & 2 & $(5.7)$ \\
\hline \multicolumn{5}{|l|}{ Other sports, no. (\%) } \\
\hline No & 20 & $(60.6)$ & 22 & $(62.9)$ \\
\hline Yes & 13 & $(39.4)$ & 13 & $(37.1)$ \\
\hline $\mathrm{BMI}, n, \mathrm{M} \pm \mathrm{SD}$ & 34 & $26.05 \pm 3.15$ & 36 & $25.58 \pm 4.19$ \\
\hline \multicolumn{5}{|l|}{ Hypercholesterolemia, no. (\%) } \\
\hline No & 29 & $(82.9)$ & 34 & $(94.4)$ \\
\hline Yes & 6 & $(17.1)$ & 2 & $(5.6)$ \\
\hline \multicolumn{5}{|l|}{ Hypercholesterolemia treatment } \\
\hline No & 31 & $(88.6)$ & 35 & $(97.2)$ \\
\hline Yes & 4 & $(11.4)$ & 1 & $(2.8)$ \\
\hline \multicolumn{5}{|l|}{ Diabetes, no. (\%) } \\
\hline No & 34 & $(97.1)$ & 36 & $(100.0)$ \\
\hline Yes & 1 & $(2.9)$ & 0 & $(0.0)$ \\
\hline \multicolumn{5}{|l|}{ Diabetes treatment } \\
\hline No & 34 & $(97.1)$ & 36 & $(100.0)$ \\
\hline Yes & 1 & $(2.6)$ & 0 & $(0.0)$ \\
\hline \multicolumn{5}{|l|}{ Hyperuricaemia, no. (\%) } \\
\hline No & 35 & $(100.0)$ & 36 & $(100.0)$ \\
\hline \multicolumn{5}{|l|}{ Tendon involvement, no. (\%) } \\
\hline Lateral & 31 & $(88.6)$ & 29 & $(80.6)$ \\
\hline Medial & 4 & $(11.4)$ & 7 & $(19.4)$ \\
\hline \multicolumn{5}{|l|}{ Location, no. (\%) } \\
\hline Left & 9 & $(25.7)$ & 15 & 41.7 \\
\hline Right & 26 & $(74.3)$ & 21 & 58.3 \\
\hline \multicolumn{5}{|l|}{ Neovascularization, no. (\%) } \\
\hline No neovascularization & 7 & $(20.0)$ & 6 & $(16.7)$ \\
\hline Neovessels on the tendon surface & 2 & $(5.7)$ & 3 & $(8.3)$ \\
\hline Intratendinous neovessels & 26 & $(74.3)$ & 27 & $(75.0)$ \\
\hline \multicolumn{5}{|l|}{ Echotexture grading scale, no. (\%) } \\
\hline Normal & 3 & $(8.6)$ & 1 & $(2.8)$ \\
\hline Hypoechogenicity $<1 / 3$ of the tendon & 2 & $(5.7)$ & 4 & $(11.1)$ \\
\hline Hypoechogenicity $>1 / 3$ and $<2 / 3$ & 10 & $(28.6)$ & 11 & $(30.6)$ \\
\hline Hypoechogenicity $>2 / 3$ & 15 & $(42.9)$ & 17 & $(47.2)$ \\
\hline Partial-thickness tear & 5 & $(14.3)$ & 3 & $(8.3)$ \\
\hline
\end{tabular}


Table 1 Baseline sociodemographic clinical and sonographic characteristics in both groups (Continued)

\begin{tabular}{|c|c|c|c|c|}
\hline \multirow{2}{*}{$\begin{array}{l}\text { Variable } \\
\text { Age, } n, M \pm S D\end{array}$} & \multicolumn{2}{|c|}{ Lidocaine $(N=35)$} & \multicolumn{2}{|c|}{$\operatorname{PRP}(N=36)$} \\
\hline & 35 & $48.26 \pm 7.64$ & 36 & $50.80 \pm 6.73$ \\
\hline \multicolumn{5}{|l|}{ Calcifications, no. (\%) } \\
\hline No & 28 & $(80.0)$ & 32 & $(91.4)$ \\
\hline Yes & 7 & $(20.0)$ & 3 & (8.6) \\
\hline Tendon thickness, $n, M \pm S D$ & 35 & $1.16 \pm 0.21$ & 36 & $1.17 \pm 0.21$ \\
\hline $\mathrm{DASH}-\mathrm{E}, n, \mathrm{M} \pm \mathrm{SD}$ & 33 & $44.06 \pm 14.05$ & 36 & $44.74 \pm 17.09$ \\
\hline VAS-P, $n, M \pm S D$ & 35 & $5.87 \pm 1.52$ & 35 & $5.91 \pm 1.78$ \\
\hline
\end{tabular}

$B M I$ body mass index, DASH-E Spanish version of the Disabilities of the Arm, Shoulder and Hand questionnaires, $M$ mean, $S D$ standard deviation, PRP platelet-rich plasma, VAS-P visual analogue scale for pain $\backslash$

8.63 to 52.02$)$ and $20.81 \%(95 \% \mathrm{CI},-6.84$ to 48.47$)$ at 3 months.

Essentially, the time course was different between treatments. In the PRP group, the increase in the rates of patients who achieved functional improvement and pain reduction was steadier than in the lidocaine group, which reached a plateau at 3 months. Furthermore, there was no statistical evidence of a modification in treatment effects by the controlled patient's factors. When data were adjusted for confounders, both groups showed enhanced function and pain reduction over the 12-month follow-up without significant differences between PRP and lidocaine (see Fig. 2 and Table 3).

There were no differences between lidocaine and PRP in DASH-E and VAS-P over time (Fig. 3a, b).

The estimated power calculated with our current data, for small, medium and big differences for DASH-E were $0.12,0.26$ and 0.93 , respectively. Likewise, estimated powers for VAS-P were $0.18,0.81$ and 0.99 , respectively.

\section{Safety}

Twenty-three adverse events in twelve patients (six patients from each treatment group) were reported as probably $(n=22)$ or likely $(n=1)$ related to treatment. Pain and swelling were the most commonly reported in the 6 weeks following tenotomy. In addition, throughout the 12-month follow-up, a total of 593 events were reported as unlikely or not related to the treatment. They included headache, migraines, back pain, shoulder pain, gastrointestinal discomfort and allergies, with cold and flu being the most common among others.

\section{Discussion}

Our hypothesis stated that PRP could be an effective adjuvant to elbow tenotomy (medial and lateral) in patients who were recalcitrant to conservative treatment. As tenotomy is an active treatment that per se influences outcomes in tendinopathy [10], our goal was not to test PRP on its own, but to determine the clinical efficacy of the combined procedure [tenotomy + PRP]. We found that two tenotomies (one strong, approximately 20-25 penetrations and bone abrasion, and the second weaker, approximately 10 penetrations) with PRP or lidocaine were effective to reduce pain and improve function over 6 months. Moreover, the improvement was maintained at 1 year after intervention, which is beyond the limits of recurrence. However,

Table 2 Association between DASH-E and VAS-P by treatment

\begin{tabular}{|c|c|c|c|c|c|}
\hline \multirow[t]{2}{*}{ VAS-P } & \multicolumn{5}{|l|}{ DASH-E } \\
\hline & Basal & 6 weeks & 3 months & 6 months & 12 months \\
\hline Basal, $r(n)$ & $\begin{array}{l}0.28^{*}(33) \\
0.52^{*}(35)\end{array}$ & $\begin{array}{l}0.59 *(25) \\
0.26(28)\end{array}$ & $\begin{array}{l}0.42^{*}(26) \\
0.28(27)\end{array}$ & $\begin{array}{l}0.46^{*}(25) \\
0.24(25)\end{array}$ & $\begin{array}{l}0.16(25) \\
0.25(24)\end{array}$ \\
\hline 6 weeks, $r(n)$ & $\begin{array}{l}0.26(28) \\
0.34(32)\end{array}$ & $\begin{array}{l}0.70^{*}(24) \\
0.83^{*}(28)\end{array}$ & $\begin{array}{l}0.63^{*}(24) \\
0.67^{*}(25)\end{array}$ & $\begin{array}{l}0.48^{*}(22) \\
0.47^{*}(23)\end{array}$ & $\begin{array}{l}0.37(24) \\
0.45^{*}(22)\end{array}$ \\
\hline 3 months, $r(n)$ & $\begin{array}{l}0.23(27) \\
0.21(29)\end{array}$ & $\begin{array}{l}0.53^{*}(23) \\
0.60^{*}(24)\end{array}$ & $\begin{array}{l}0.74^{*}(25) \\
0.79^{*}(27)\end{array}$ & $\begin{array}{l}0.63^{*}(23) \\
0.77^{*}(23)\end{array}$ & $\begin{array}{l}0.51^{*}(23) \\
0.76^{*}(20)\end{array}$ \\
\hline 6 months, $r(n)$ & $\begin{array}{l}0.34^{*}(27) \\
0.20(27)\end{array}$ & $\begin{array}{l}0.56^{*}(22) \\
0.63^{*}(22)\end{array}$ & $\begin{array}{l}0.67^{*}(23) \\
0.84^{*}(25)\end{array}$ & $\begin{array}{l}0.94^{*}(24) \\
0.88^{*}(23)\end{array}$ & $\begin{array}{l}0.73^{*}(23) \\
0.79^{*}(21)\end{array}$ \\
\hline 12 months, $r(n)$ & $\begin{array}{l}0.11(24) \\
0.07(23)\end{array}$ & $\begin{array}{l}0.32(21) \\
0.42^{*}(18)\end{array}$ & $\begin{array}{l}0.34(22) \\
0.50^{*}(21)\end{array}$ & $\begin{array}{l}0.64^{*}(21) \\
0.63^{*}(20)\end{array}$ & $\begin{array}{l}0.91 *(24) \\
0.77^{*}(20)\end{array}$ \\
\hline
\end{tabular}

Data without italics represent lidocaine; data with italics represent PRP

$D A S H-E$ Spanish version of the Disabilities of the Arm, Shoulder and Hand questionnaires, $r$ Spearman's rank correlation coefficient, VAS- $P$ visual analogue scale for pain assessment

${ }^{*} p<0.05$

Boldface and bold italics represent statistically significative associations 
Table 3 Outcome measurements and adjusted intergroup differences

\begin{tabular}{|c|c|c|c|c|c|}
\hline \multirow[b]{2}{*}{ Outcome } & \multicolumn{3}{|c|}{ Unadjusted change } & \multicolumn{2}{|l|}{ Odds ratio $(95 \% \mathrm{Cl})$} \\
\hline & Lidocaine & PRP & $\begin{array}{l}\text { Treatment-time measurement } \\
\text { interaction ( } p \text { value) }\end{array}$ & Unadjusted & Adjusted $^{a}$ \\
\hline $\begin{array}{l}\% \text { meeting DASH-E improvement, } \\
\text { no./total no. (\%) }\end{array}$ & & & .307 & & \\
\hline $6-7$ weeks & $11 / 24(45.83)$ & 10/28 (35.71) & & 1.37 (0.31 to 6.04$)$ & $1.33(0.31$ to 5.71$)$ \\
\hline 3 months & 20/24 (83.33) & $17 / 29(58.62)$ & & 4.73 (0.86 to 26.08) & 5.67 (0.98 to 32.74$)$ \\
\hline 6 months & $18 / 25(72.00)$ & 19/26 (73.08) & & 0.90 (0.17 to 4.60$)$ & 1.15 (0.22 to 5.95$)$ \\
\hline 12 months & $17 / 24(70.83)$ & $19 / 25(76.00)$ & & 0.71 (0.13 to 3.84) & 0.91 (0.17 to 4.88$)$ \\
\hline $\begin{array}{l}\% \text { meeting VAS-P improvement, } \\
\text { no./total no. (\%) }\end{array}$ & & & .105 & & \\
\hline 6-7 weeks & 19/30 (63.33) & 13/31 (41.94) & & 2.42 (0.67 to 8.76$)$ & 2.67 (0.74 to 9.68$)$ \\
\hline 3 months & $22 / 29(75.86)$ & $16 / 28(57.14)$ & & 2.78 (0.68 to 11.38$)$ & 2.92 (0.71 to 12.04$)$ \\
\hline 6 months & $21 / 29(72.41)$ & $22 / 26(84.62)$ & & 0.48 (0.10 to 2.37$)$ & 0.50 (0.10 to 2.48$)$ \\
\hline 12 months & 19/25 (76.00) & 20/22 (90.91) & & 0.3580 .06 to 2.51$)$ & 0.42 (0.06 to 3.03$)$ \\
\hline Median DASH-E scores (IQR) & & & .429 & & \\
\hline 6-7 weeks & $30.17(30.0)$ & $40.59(34.86)$ & & NA & NA \\
\hline 3 months & $16.49(29.04)$ & $31.73(34.94)$ & & NA & NA \\
\hline 6 months & $12.93(28.44)$ & $14.25(26.66)$ & & NA & NA \\
\hline 12 months & $7.50(31.15)$ & $9.17(24.17)$ & & NA & NA \\
\hline Median VAS-P scores (IQR) & & & .441 & & \\
\hline 6-7 weeks & $4.00(3.00)$ & $4.25(4.75)$ & & NA & NA \\
\hline 3 months & $2.00(2.50)$ & $4.00(5.00)$ & & NA & NA \\
\hline 6 months & $2.00(4.40)$ & $2.00(3.50)$ & & NA & NA \\
\hline 12 months & $2.00(3.50)$ & $2.00(4.00)$ & & NA & NA \\
\hline
\end{tabular}

Cl confidence interval, DASH-E Spanish version of the Disabilities of the Arm, Shoulder and Hand questionnaires, IQR interquartile range, NA not applicable, no number, VAS- $P$ visual analogue scale for pain assessment

${ }^{\mathrm{a}} \%$ meeting DASH-E and VAS-P improvement were adjusted for the degree of vascularization before treatment

we did not find any clinical differences between the effects of the adjuvants, PRP and lidocaine, in pain reduction or in functional recovery at 6 or 12 months.

According to a recent meta-analysis [14] (including 12 studies of painful lateral epicondylopathy), an effect size of 0.57 favoured PRP treatment. Thus, our trial was underpowered, as a sample size of 73 patients per group would be necessary to detect any significant difference in clinical outcomes. According to these data, most studies in the literature, except one study [23] examining PRP in elbow epicondylopathy, are underpowered. Moreover, the probability of superiority and the number needed to treat varied depending on the comparator [24].

In fact, PRPs have been compared with various injectables, including autologous blood [25-27], saline [2830 ] or corticosteroids [22, 28, 29, 31], using different treatment protocols. In addition to the choice of the comparator, the use (or not) of tenotomy or an alternative milder peppering technique (approximately five perforations) can yield to different temporal differences in PRP efficacy. For example, Peerbooms et al. [22] and Liebiedzinsky et al. [31] used a peppering technique and demonstrated that L-PRP was superior to corticosteroids at 12 months but not at the 3- and 6-month follow-ups, corroborating data from other studies with only a 3 -month follow-up [28]. On the other hand, other studies with small samples per group (20-25 patients) failed to find differences between saline and L-PRP at three months [28] or between saline and pure PRP at 6 months or 12 months [30]. The latter study involved patients with recent epicondylitis. Likewise, when autologous blood was used as a comparator, there were no relevant differences in primary outcomes over a 6-month period $[25,26]$. According to the estimated potency using our clinical data, our results are not conclusive due to an inadequate sample number. However, all these studies are valuable as they contribute to stronger findings through meta-analyses and umbrella reviews.

Although we failed to show differences between PRP and lidocaine, our results do match the promising results observed in a previous multicentre study comparing L-PRP with anaesthetics in lateral elbow tendinopathy [23]. Weak evidence (retrospective case-controlled study with few patients) for better outcomes with pure PRP at 6 and 12 


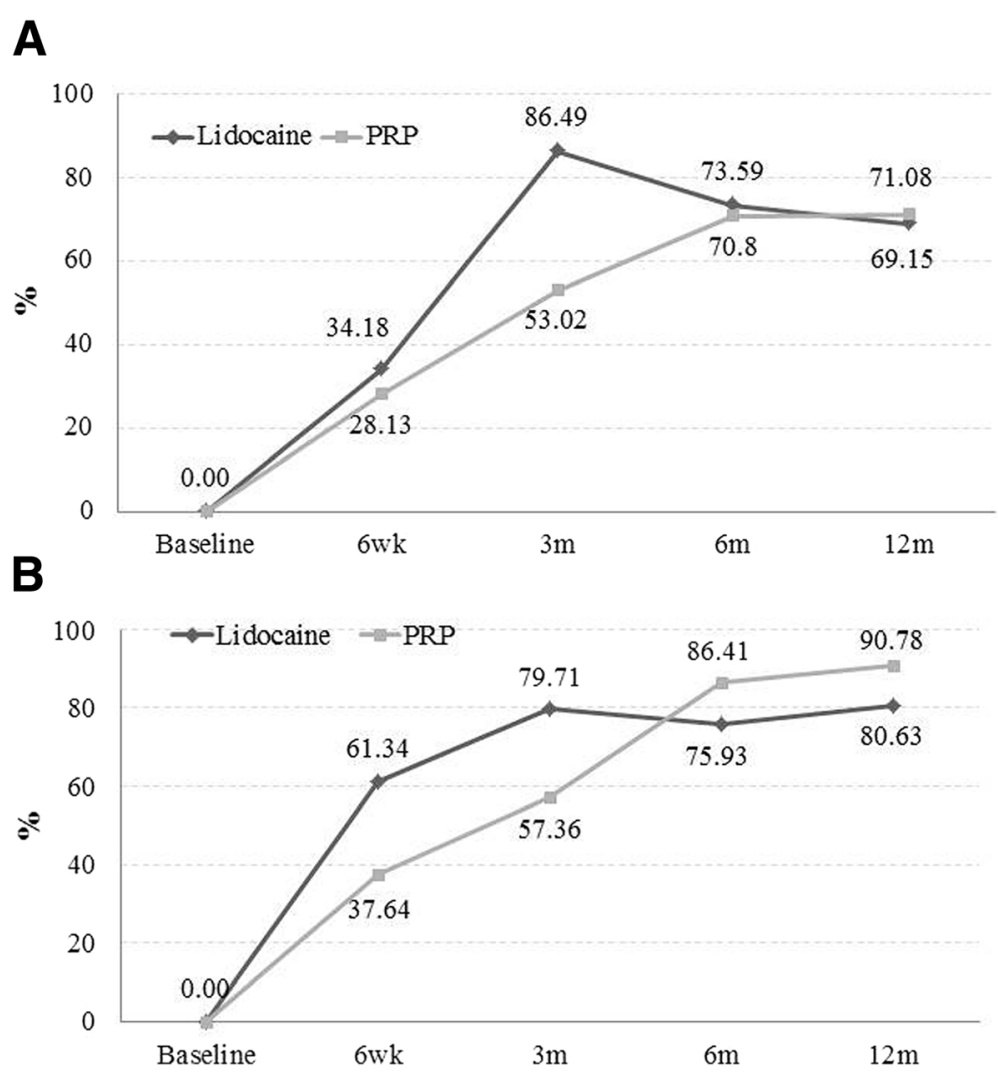

Fig. 2 Percentage of patients with an improvement exceeding 25\% reduction in DASH-E (a) and in VAS-P (b) over 1-year follow-up, after adjusting data for baseline vascularization. DASH-E, Spanish version of the Disabilities of the Arm, Shoulder and Hand questionnaires; VAS-P, visual analogue scale for pain; $m$, month

months compared to bupivacaine has been reported [32]. In contrast, Palacio et al. [29] did not find any difference at 3 or 6 months when comparing PRP with anaesthetics or corticosteroids in untreated patients after blind $3 \mathrm{~mL}$ injections. To the best of our knowledge, only the three above referenced studies [23, 29,32] compared injections of PRP versus anaesthetics. However, the number of treatments, PRP formulation and injected volume and intensity of tenotomy differed among studies.

We chose lidocaine as a comparator because it is our routine practice to enhance patient and physician comfort during the procedure. Indeed, tenotomy is very painful. Whether lidocaine is an active or placebo control in this context is controversial. Actually, there is general concern regarding the intratendinous administration of anaesthetics, because it might compromise cell viability [33]. Similarly, administering anaesthetics peripherally, which is common clinical practice in order to reduce pain during intervention, could damage cells from the extrinsic compartment that participate in early extrinsic repair [34]. Furthermore, the volume of lidocaine delivered in the peritendon of the common extensor origin varied between studies and was as high as $10-15 \mathrm{~mL}$ lidocaine $(10 \mathrm{mg} / \mathrm{mL})$ [28]. Lidocaine may induce cytotoxicity (apoptosis and/or necrosis) through mitochondrial insults mediated by intracellular radical oxygen species (ROS), by specific mitogen-activated protein kinases (MAPKs) (i.e., ERK1/2, JNK and p38) and by caspase-3/7 in vitro [34]. However, differences in cytotoxic vulnerability between healthy and tendinopathic cells in the intrinsic and extrinsic compartments and whether PRP reinforces lidocaine cytotoxicity or not have not been explored yet. Despite all these negative in vitro data related to lidocaine, our patients in the lidocaine group showed a similar safety profile to that of patients receiving PRP.

In addition to the choice of active (blood, corticosteroids) or placebo comparators (saline), the efficacy of PRP in tendon interventions may be strongly linked to other factors that can have biological/clinical relevance, e.g., first, whether the intervention is echo-guided to control the spatial delivery of the product, i.e., peri-tendinous, intratendinous or both, and second, the intensity of the tenotomy procedure, which can vary from 5 to 50 penetrations. Finally, the composition of PRPs varies between studies in terms of product composition and application protocol. According to the Platelet Physiology Subcommittee of the Scientific and Standardization Committee of the International Society on Thrombosis and Haemostasis (ISTH), 

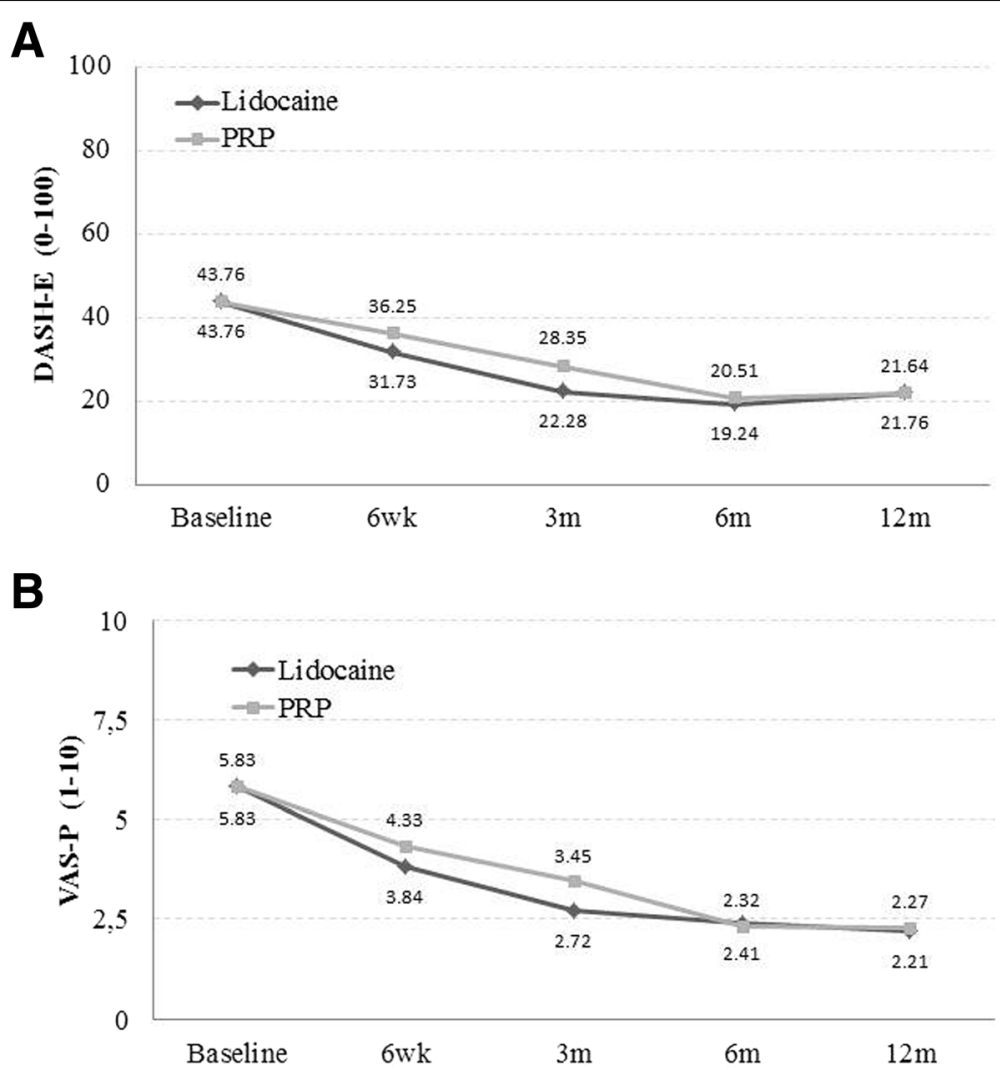

Fig. 3 Changes of DASH-E (a) and VAS-P (b) over time, after adjusting data for baseline DASH-E and VAS-P scores, vascular status and hypercholesterolemia. DASH-E, Spanish version of the Disabilities of the Arm, Shoulder and Hand questionnaires; PRP, platelet-rich plasma; VAS-P, visual analogue scale for pain; $m$, month

the plasma product used in this trial was recalcified at 4.5 $\mathrm{mL}(\times 2)$ PRP IIA1 [35]. The biological properties of this PRP product regarding inflammation and angiogenesis [11], cell migration, proliferation and anabolic effects have been fully examined in tenocyte cultures $[12,17]$, but they used frozen-thawed allogeneic PRP.

Compared to other clinical studies, we used a relatively high volume $(4-5 \mathrm{~mL}$ versus $1-3 \mathrm{~mL})$. This high volume implies diffusion of PRP away from the injection sites, reaching adjacent peritendinous tissues, including the subcutaneous fat and peritenon (epitenon) [36, 37]. Actually, activation of the extrinsic compartment (with a higher number of cells than the proper tendon) is also needed to drive healing through the complementary actions of intrinsic and extrinsic cells. However, how the extrinsic and intrinsic compartments interact in the failed healing response is unknown and whether PRP (or other regenerative products) should be delivered intraor peritendinously or both and their interaction with local anaesthetics should be investigated. According to recent data [18], peritendinous delivery of PRP in enthesopathies was effective in pain reduction and produced better results than injections within the proper tendon in proper tendinopathies.
Our protocol also differed from those that published the number of interventions. In fact, consistent with the idea that tendinopathy is acquired through many years and the fact that a unique intervention is unlikely to change the tendon structure and fate of tendinopathy, we have performed two interventions (tenotomies). Other authors $[25,30,38]$ repeated the injections $2-4$ weeks apart. While there is no evidence on the benefits of the second injection in epicondylopathy [39], a second injection enhanced outcomes in a randomized prospective study in patellar tendinopathy [40]. Hopefully, in the future, interventions would be tailored to patient subgroups with different tendon needs.

Our study has several limitations. First, the diagnosis was merely clinical based on clinical signs and local pain. Second, whether PRP is superior to lidocaine in this context remains unsolved in this trial, in part because of the large amount of missing data not attributable to lost patients, but for other reasons. We did not perform telephone reminders regarding patient appointments and did not control if those patients were examined in the sonographic service attending their orthopaedist appointment. In fact, some of them missed their clinical appointment because of long waiting times. Moreover, 
some DASH-E questionnaires were incompletely filled and could not be computed.

Third, the lack of a dry tenotomy group in our trial means that we cannot, with certainty, rule out the effect of "dry" tenotomy as the main driver of therapeutic benefits. Moreover, the effects of PRP or lidocaine without tenotomy remain to be elucidated. Lastly, DASH-E is the sole functional score validated in Spanish, but it has important limitations for assessing elbow function, as other upper limb pathologies result in artefacts in DASH-E scores.

\section{Conclusions}

The intention-to-treat analysis revealed that a double tenotomy, with lidocaine or PRP as adjuvants, is effective in producing a clinically significant reduction in DASH-E and VAS-P scores in a cohort of patients with recalcitrant elbow tendinopathy. In up to $70 \%$ of the cases, tenotomy can succeed when conservative therapies have already failed, and both adjuvants appear to be of comparable efficacy with this limited sample size. We thus recommend "humid" tenotomy with PRP/lidocaine as an important second line option in patients in whom conservative physical therapy has failed. As always, "the devil is in the details"; thus, we have focused here on describing and discussing the details of our protocol that could have clinical consequences.

\section{Abbreviations}

BMI: Body mass index; Cl: Confidence interval; DASH-E: Spanish version of the Disabilities of the Arm, Shoulder and Hand questionnaires; ECRB: Extensor carpi radialis brevis; HUC: Hospital Universitario Cruces; IQR: Interquartile range; ISTH: International Society on Thrombosis and Haemostasis; LPRP: Leukocyte- rich, platelet-rich plasma; M: Mean; MAPKs: Mitogenactivated protein kinases; NA: Not applicable; NSAIDs: Non-steroidal antiinflammatory drugs; PRP: Platelet-rich plasma; SD: Standard deviation; US: Ultrasound; VAS-P: Visual analogue scale for pain

\section{Acknowledgements}

The authors wish to thank Nuria Zuazo for monitoring data acquisition and Mercedes Moreno and Julio del Bosque for nursing assistance.

\section{Funding}

This work was supported by "Instituto de Salud Carlos III", ISCIII (grant number PI13/01707 to I.A.) co-financed by FEDER funds.

\section{Availability of data and materials}

The data from the current trial are available from the corresponding author on reasonable request

\section{Authors' contributions}

$J I M, L A, J M, L A, G$ and IA contributed to the conceptualization. GG, PB and IA contributed to the methodology. PB and GG contributed to the formal analysis. JIM, LA, JM, LMA, GI, IG and IA contributed to the investigation. JIM, $L A, J M, L M A, G I, I G$ and IA contributed the resources. PB and IA contributed to the data curation. JIM, PB, and IA contributed to the writing and preparation of the original draft. JIM, LA, PB, GG and IA contributed to the writing, reviewing and editing of the manuscript. Supervision was done by IA and GG. IA contributed to the project administration., IA and JIM contributed to the funding acquisition. All authors read and approved the final manuscript.

\section{Ethics approval and consent to participate}

This study was approved by the Ethics Committee of Hospital Universitario Cruces (No. CEIC 13/04). All enrolled patients provided written informed consent. The study protocol was registered at ClinicalTrials.gov (identifier NCT01945528) EUDRACT No.2013-000478-32.

All procedures were in accordance with the ethical standards of the institutional and/or national research committee and with the 1964 Helsinki

Declaration and its later amendments or comparable ethical standards.

\section{Consent for publication}

All participants gave their written consent for publication

\section{Competing interests}

The authors declare no conflicts of interest.

\section{Publisher's Note}

Springer Nature remains neutral with regard to jurisdictional claims in published maps and institutional affiliations.

\section{Author details}

${ }^{1}$ Interventional Sonography, Department of Radiology, Cruces University Hospital, Barakaldo, Spain. ${ }^{2}$ Regenerative Medicine, BioCruces Health Research Institute, Cruces University Hospital, 48903 Barakaldo, Spain. ${ }^{3}$ Department of Orthopaedic Surgery, Cruces University Hospital, Barakaldo, Spain. ${ }^{4}$ Primary Care Research Unit of Bizkaia (Basque Healthcare Service), BioCruces Health Research Institute, Bilbao, Spain.

Received: 16 November 2018 Accepted: 12 April 2019

Published online: 23 April 2019

\section{References}

1. Degen RM, Conti MS, Camp CL, Altchek DW, Dines JS, Werner BC. Epidemiology and disease burden of lateral epicondylitis in the USA: analysis of 85,318 patients. HSS J. 2018;14(1):9-14.

2. Shiri $R$, Viikari-Juntura $E$, Varonen $H$, Heliövaara M. Prevalence and determinants of lateral and medial epicondylitis: a population study. Am J Epidemiol. 2006;164(11):1065-74.

3. Pitzer ME, Seidenberg PH, Bader DA. Elbow tendinopathy. Med Clin North Am. 2014;98(4):833-49.

4. Descatha A, Albo F, Leclerc A, Carton M, Godeau D, Roquelaure Y, et al. Lateral epicondylitis and physical exposure at work? a review of prospective studies and meta-analysis. Arthritis Care Res (Hoboken). 2016;68(11):1681-7.

5. Spang C, Alfredson H. Richly innervated soft tissues covering the superficial aspect of the extensor origin in patients with chronic painful tennis elbow Implication for treatment? J Musculoskelet Neuronal Interact. 2017;17(2):97-103.

6. Abate M, Salini V, Schiavone C, Andia I. Clinical benefits and drawbacks of local corticosteroids injections in tendinopathies. Expert Opin Drug Saf. 2017:16(3):341-9.

7. Gaspar MP, Motto MA, Lewis S, Jacoby SM, Culp RW, Lee Osterman A. Platelet-rich plasma injection with percutaneous needling for recalcitrant lateral epicondylitis: comparison of tenotomy and fenestration techniques. Orthop J Sports Med. 2017;5(12):2325967117742077.

8. Hammerman M, Aspenberg P, Eliasson P. Microtrauma stimulates rat Achilles tendon healing via an early gene expression pattern similar to mechanical loading. J Appl Physiol (1985). 2014;116(1):54-60.

9. Jacobson JA, Kim SM, Brigido MK. Ultrasound-guided percutaneous tenotomy. Semin Musculoskelet Radiol. 2016;20(5):414-21.

10. Mattie R, Wong J, McCormick Z, Yu S, Saltychev M, Laimi K. Percutaneous needle tenotomy for the treatment of lateral epicondylitis: a systematic review of the literature. PM R. 2017;9(6):603-11.

11. Andia I, Rubio-Azpeitia E, Maffulli N. Platelet-rich plasma modulates the secretion of inflammatory/angiogenic proteins by inflamed tenocytes. Clin Orthop Relat Res. 2015;473(5):1624-34.

12. Rubio-Azpeitia E, Sánchez P, Delgado D, Andia I. Three-dimensional plateletrich plasma hydrogel model to study early tendon healing. Cells Tissues Organs. 2015;200(6):394-404

13. Fitzpatrick J, Bulsara M, Zheng MH. The effectiveness of platelet-rich plasma in the treatment of tendinopathy: a meta-analysis of randomized controlled clinical trials. Am J Sports Med. 2017;45(1):226-33.

14. Miller LE, Parrish WR, Roides B, Bhattacharyya S. Efficacy of platelet-rich plasma injections for symptomatic tendinopathy: systematic review and 
meta-analysis of randomised injection-controlled trials. BMJ Open Sport Exerc Med. 2017:3(1):e000237.

15. Chen $X$, Jones IA, Park $C$, Vangsness $C T$ Jr. The efficacy of platelet-rich plasma on tendon and ligament healing: a systematic review and metaanalysis with bias assessment. Am J Sports Med. 2018;46(8):2020-32.

16. Saltychev M, Laimi K, Virolainen P, Fredericson M. Effectiveness of platelet rich plasma in the treatment of lateral epicondylitis-a systematic review and metaanalysis. PMR+. 2018;1 (1):7-16.

17. Rubio-Azpeitia E, Bilbao AM, Sánchez P, Delgado D, Andia I. The properties of 3 different plasma formulations and their effects on tendinopathic cells. Am J Sports Med. 2016;44(8):1952-61.

18. Papalia R, Zampogna B, Vadala G, et al. Are platelet rich plasma injections more effective in tendinopathy or enthesopathy? J Pain Relief. 2017;6(3):1-5.

19. Martin Jl, Merino J, Atilano L, Areizaga LM, Gomez-Fernandez MC, BurgosAlonso N, et al. Platelet-rich plasma (PRP) in chronic epicondylitis: study protocol for a randomized controlled trial. Trials. 2013;14:410.

20. Dohan Ehrenfest DM, Andia I, Zumstein MA, Zhang CQ, Pinto NR, Bielecki T. Classification of platelet concentrates (Platelet-Rich Plasma-PRP, Platelet-Rich Fibrin-PRF) for topical and infiltrative use in orthopedic and sports medicine: current consensus, clinical implications and perspectives. Muscles Ligaments Tendons J. 2014:4(1):3-9 eCollection 2014 Jan. Review.

21. Franchignoni F, Vercelli S, Giordano A, Sartorio F, Bravini E, Ferriero G. Minimal clinically important difference of the disabilities of the arm, shoulder and hand outcome measure (DASH) and its shortened version (QuickDASH). J Orthop Sports Phys Ther. 2014;44(1):30-9.

22. Peerbooms JC, Sluimer J, Bruijn DJ, Gosens T. Positive effect of an autologous platelet concentrate in lateral epicondylitis in a double-blind randomized controlled trial: platelet-rich plasma versus corticosteroid injection with a 1-year follow-up. Am J Sports Med. 2010;38(2):255-62.

23. Mishra AK, Skrepnik NV, Edwards SG, Jones GL, Sampson S, Vermillion DA, et al. Platelet-rich plasma significantly improves clinical outcomes in patients with chronic tennis elbow: a double-blind, prospective, multicenter, controlled trial of 230 patients. Am J Sports Med. 2014;42(2):463-71.

24. Tsikopoulos K, Tsikopoulos I, Simeonidis E, Papathanasiou E, Haidich AB, Anastasopoulos N, et al. The clinical impact of platelet-rich plasma on tendinopathy compared to placebo or dry needling injections: a metaanalysis. Phys Ther Sport. 2016;17:87-94.

25. Creaney L, Walla A, Curtis M, Connell D. Growth factor-based therapies provide additional benefit beyond physical therapy in resistant elbow tendinopathy: a prospective, double-blind, randomised trial of autologous blood injections versus platelet-rich plasma injections. Br J Sports Med. 2011:45(12):966-71.

26. Thanasas C, Papadimitriou G, Charalambidis C, Paraskevopoulos I, Papanikolaou A. Platelet-rich plasma versus autologous whole blood for the treatment of chronic lateral elbow epicondylitis: a randomized controlled clinical trial. Am J Sports Med. 2011;39(10):2130-4.

27. Raeissadat SA, Rayegani SM, Hassanabadi H, Rahimi R, Sedighipour L, Rostami K. Is platelet-rich plasma superior to whole blood in the management of chronic tennis elbow: one year randomized clinical trial. BMC Sports Sci Med Rehabil. 2014;6(1):12.

28. Krogh TP, Fredberg U, Stengaard-Pedersen K, Christensen R, Jensen P, Ellingsen $T$. Treatment of lateral epicondylitis with platelet-rich plasma, glucocorticoid, or saline: a randomized, double-blind, placebo-controlled trial. Am J Sports Med. 2013;41(3):625-35.

29. Palacio EP, Schiavetti RR, Kanematsu M, Ikeda TM, Mizobuchi RR, Galbiatti JA Effects of platelet-rich plasma on lateral epicondylitis of the elbow: prospective randomized controlled trial. Rev Bras Ortop. 2016;51 (1):90-5.

30. Montalvan B, Le Goux P, Klouche S, Borgel D. Hardy P5, Breban M. Inefficacy of ultrasound-guided local injections of autologous conditioned plasma for recent epicondylitis: results of a double-blind placebo-controlled randomized clinical trial with one-year follow-up. Rheumatology (Oxford). 2016:55(2):279-85.

31. Lebiedziński R, Synder M, Buchcic Polguj M, Grzegorzewski A, Sibiński M. A randomized study of autologous conditioned plasma and steroid injections in the treatment of lateral epicondylitis. Int Orthop. 2015;39(11):2199-203.

32. Behera P, Dhillon M, Aggarwal S, Marwaha N, Prakash M. Leukocyte-poor platelet-rich plasma versus bupivacaine for recalcitrant lateral epicondylar tendinopathy. J Orthop Surg (Hong Kong). 2015;23(1):6-10.

33. Wang WZ, Fang XH, Williams SJ, Stephenson LL, Baynosa RC, Khiabani KT, et al. Lidocaine-induced ASC apoptosis (tumescent vs. local anesthesia). Aesthetic Plast Surg. 2014;38(5):1017-23.
34. Sung CM, Hah YS, Kim JS, Nam JB, Kim RJ, Lee SJ, et al. Cytotoxic effects of ropivacaine, bupivacaine, and lidocaine on rotator cuff tenofibroblasts. Am J Sports Med. 2014;42(12):2888-96.

35. Harrison P. Subcommittee on Platelet Physiology. The use of platelets in regenerative medicine and proposal for a new classification system: guidance from the SSC of the ISTH. J Thromb Haemost. 2018. https://doi. org/10.1111/jth. 14223 .

36. Loftus ML, Endo Y, Adler RS. Retrospective analysis of postinjection ultrasound imaging after platelet-rich plasma or autologous blood: observational review of anatomic distribution of injected material. AJR Am J Roentgenol. 2012;199(4):W501-5.

37. Park GY, Kwon DR, Cho HK, Park J, Park JH. Distribution of platelet-rich plasma after ultrasound-guided injection for chronic elbow tendinopathies. J Sports Sci Med. 2017;16(1):1-5.

38. Stenhouse G, Sookur P, Watson M. Do blood growth factors offer additional benefit in refractory lateral epicondylitis? A prospective, randomized pilot trial of dry needling as a stand-alone procedure versus dry needling and autologous conditioned plasma. Skeletal Radiol. 2013;42(11):1515-20.

39. Glazmann MC, Audigé L. Platelet-rich plasma for chronic lateral epicondylitis: Is one injection sufficient? Arch Orthop Trauma Surg. 2015; 135(12):1637-45.

40. Zayni R, Thaunat M, Fayard JM, Hager JP, Carrillon Y, Clechet J, et al. Platelet-rich plasma as a treatment for chronic patellar tendinopathy: comparison of a single versus two consecutive injections. Muscles Ligaments Tendons J. 2015;5(2):92-8.

\section{Ready to submit your research? Choose BMC and benefit from:}

- fast, convenient online submission

- thorough peer review by experienced researchers in your field

- rapid publication on acceptance

- support for research data, including large and complex data types

- gold Open Access which fosters wider collaboration and increased citations

- maximum visibility for your research: over $100 \mathrm{M}$ website views per year

At BMC, research is always in progress.

Learn more biomedcentral.com/submissions 